\title{
ROTATION OF PRE-MAIN SEQUENCE STARS FROM HIGH S/N SPECTROSCOPY
}

\author{
J. Bouvier \\ Institut d'Astrophysique \\ 98 bis bd Arago \\ F-75014 Paris
}

\begin{abstract}
Until 1980, only a handful of low-mass, active pre-main sequence (pms) stars had known rotation velocities (vsini) $/ 1 /$. Since then, increasingly sensitive detectors coupled to large telescopes led to high-resolution (a few $10^{4}$ ) spectroscopic studies of these faint stars $\left(\mathrm{m}_{v}=10-13\right)$, with $\mathrm{S} / \mathrm{N}$ ratio of the order of 100 . The measurement of $v$ sini for large samples of pms stars that resulted brought new insights on various pressing questions related to stellar formation and early stellar evolution : how do the rotation rates of pms stars compare with those expected from models of stellar formation? how does the stellar angular momentum change during pms evolution? is pms activity linked with rotation as would be expected if activity were triggered by magnetic processes?
\end{abstract}

\section{STELLAR ROTATION OF LOW-MASS YOUNG STARS}

Vogel and Kuhi (1981) /2/ obtained mostly vsini upper limits of $25-35 \mathrm{~km} . \mathrm{s}^{-1}$ for a sample of pms stars they investigated spectroscopically. That low-mass pms stars were rotating far below their break-up velocity $\left(\simeq 300 \mathrm{~km} . \mathrm{s}^{-1}\right)$ led them to conclude that most of the angular momentum presumably contained in the inital interstellar gas cloud had been removed before the star became visible at optical wavelengths.

Bouvier et al. (1986) /3/ measured the rotation rates of 28 low-mass pms stars. While confirming previous results of Vogel and Kuhi, the low detection threshold (a few $\mathrm{km} . \mathrm{s}^{-1}$ ) of CORAVEL, a cross-correlating spectrograph, allowed us to derive the vsini distribution of the sample, with vsini ranging from 8 to $73 \mathrm{~km} . \mathrm{s}^{-1}$, including no upper limits. Activity-rotation relations were then searched for in pms stars (see /4,5/).

Independently, Hartmann et al. (1986) /6/ applied cross-correlation analysis on high-quality spectrograms of 50 pms stars to measure vsini with a detection threshold of $10 \mathrm{~km} \cdot \mathrm{s}^{-1}$, leaving undertermined the rotation rates of $20 \%$ of their sample.

At the same time, the rotation rates of low-mass stars in several young clusters were obtained by Stauffer et al. $(1984,1985) / 7,8 /$, with a detection threshold of $10 \mathrm{~km} . \mathrm{s}^{-1}$.

These studies offer an observational sampling of the rotation of low-mass stars at various ages that constrains models such as Endal and Sofia's (1981) /9/ which describes the rotational evolution of a $1 \mathrm{M}_{\odot}$ star during the pms phase and up to the Sun's age. Figure 1 shows solutions of this model compared to the observational constraints. Starting from $10^{7}$ years, the surface rotation first increases as the result of stellar contraction and because a radiative core develops in the initially completely convective star. Then, 
assuming magnetic braking acts to remove angular momentum from the outer convective zone, the surface velocity starts decreasing as the star approaches the main-sequence. Model calculations of the transfer of angular momentum inside the star predict that, as the star reaches the main-sequence, the outer convective zone uncouples from the rapidly rotating radiative core and is abruptly slowed down. Although the observed trends (pms spin-up and ms spin-down) are accounted for by the model, quantitative disagreements remain. Rapid rotators in the $\alpha$ Persei cluster suggest that the pms spin-up is larger than that predicted by the model. Also, the early main-sequence braking seems to act on a time-scale much shorter than the model predicts. Several assumptions of the model may be responsible for these disagreements. New models which investigate various braking laws for stars of various masses are being currently developped by the Sofia group at Yale University and may eventually provide a better account of the observations. Ultimately, such models should enlight how angular momentum is lost during early stellar evolution (winds, magnetic fields) and will also be helpful to estimate the amount to which internal rotation may influence the depletion of light elements as the star evolves.

The observed widening of the $v$ sini distribution from the pms stars to the $\alpha$ Persei cluster (see Fig.1) remains puzzling. Although several suggestions have been made to account for the existence of slow-rotators in the young clusters (age spread $/ 7 /$, spread of initial angular momenta $/ 3 /$, rotation-independent braking law $/ 13 /$ ) further high resolution, high $\mathrm{S} / \mathrm{N}$ spectroscopic studies are needed to settle the true vsini distribution of rotators having $v \sin i$ upper limits of $10 \mathrm{~km} . \mathrm{s}^{-1}$. Pertinent observations are planned for 50

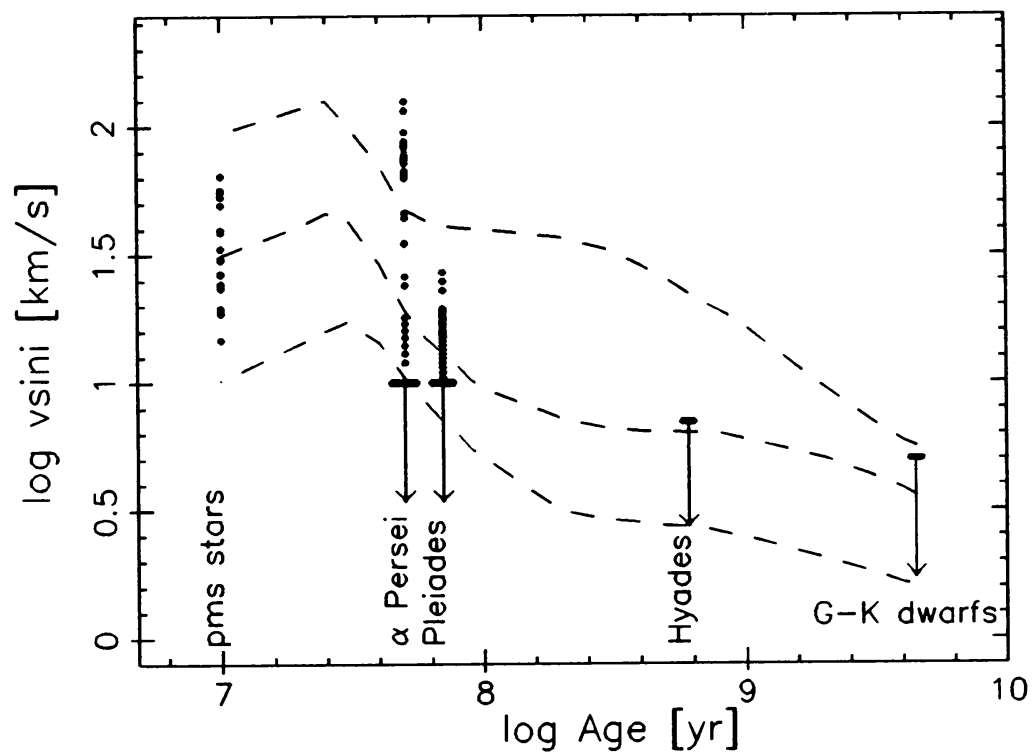

Figure 1: The distribution of surface rotational velocities of low-mass stars as a function of age. References: pms stars $\left(M \leq 1.2 M_{\odot}\right) / 3 /, \alpha$ Persei and Pleiades G0-K2 stars $/ 10 /$, Hyades $G 0-K 2$ stars $/ 11 /, G-K$ dwarfs $/ 12 /$. The vsini's of pms stars ( $10^{6}$ years) have been projected to $10^{7}$ years assuming solid-body rotation (dots). Vertical arrows denote upper limits on vsini : in $\alpha$ Persei and in Pleiades clusters, approximately half of the stars in the considered mass range have vsini $\leq 10 \mathrm{~km} . \mathrm{s}^{-1}$. For these clusters, the vsini's have been grouped in $10 \mathrm{~km} . \mathrm{s}^{-1}$-wide bins (dots). The dashed curves show solutions of Endal and Sofia's model for different amounts of initial rotation (see text). 
pms, $\alpha$ Persei and Pleiades slow rotators. Also, Lithium abundances of the slow and rapid rotators in the young clusters should be measured to explore a possible age spread.

\section{PROSPECTS}

\subsection{Doppler Imaging}

The surface of several active pms stars are believed to be covered with spots $/ 5,14,15 /$. Up to now, the spots properties were derived, with large uncertainties, from photometric observations. From now on, the new CCD camera coupled with the CES at ESO $1.4 \mathrm{~m}$ telescope provides high enough S/N (about 100) at a spectral resolution of $7.10^{4}$ to start Doppler imaging the surface of the brightest active pms stars. Long-term observations dedicated to the study of spots (growth and decay, activity cycles, latitudinal migration $\rightarrow$ surface differential rotation) should bring strong constraints on stellar dynamo models.

\subsection{Accretion disks}

Recent observational and theoretical studies have strengthened the belief that young stellar objects have circumstellar disks (e.g. /16/ and references therein). The inner parts of Keplerian disks, which are thought to radiate mostly in the near-IR range (see /17/), are expected to rotate at a few hundred $\mathrm{km} . \mathrm{s}^{-1}$, i.e., much more rapidly than the stellar photosphere. Forthcoming observations of pms disk-candidates with IRSPEC, a newly available near-IR spectrograph at the $3.6 \mathrm{~m}$ ESO telescope, will aim at testing this expectation.

\section{References}

1. Herbig, G. H., 1957, Astrophys. J., 125, 612

2. Vogel, S. N., Kuhi, L. V., 1981, Astrophys. J., 245, 960

3. Bouvier, J., Bertout, C., Benz, W., Mayor, M., 1986, Astron. Astrophys., 165, 100

4. Bouvier, J., 1987, in Molecular Clouds and Protostars, Collège de France, Paris, ed: T. Montmerle and C. Bertout, CEA Saclay: 1987, in press.

5. Bouvier, J., 1987, Thesis, Paris

6. Hartmann, L., Hewett, R., Stahler, S., Mathieu, R. D., 1986, Astrophys. J., 309, 275

7. Stauffer, J. R., Hartmann, L., Soderblom, D. R., Burnham, N., 1984, Astrophys. J., 280, 202

8. Stauffer, J. R., Hartmann, L., Burnham, N., 1985, Astrophys. J., 289, 247

9. Endal, A. S., Sofia, S., 1981, Astrophys. J., 243, 625

10. Stauffer, J. R., Hartmann, L., 1986, Publ. Astron. Soc. Pac., 98, 1233

11. Benz, W., Mayor, M., Mermilliod, J. C., 1984, Astron. Astrophys., 138, 93

12. Gray, D. F., 1982, Astrophys. J., 261, 259

13. Stauffer, J. R., Hartmann, L., 1987, Astrophys. J., 318, 337

14. Bouvier, J., Bertout, C., Bouchet, P., 1986, Astron. Astrophys., 158, 149

15. Vrba, F. J., Rydgren, A. E., Chugainov, P. F., Shakovskaya, N. I., Zak, D. S., 1986, Astrophys. J., 306, 199

16. Bertout, C., 1986, Proc. of I.A.U. Symp. no.122, "Circumstellar Matter", Heidelberg, June 1986, ed. I. Appenzeller and C. Jordan, Reidel: 1987

17. Bertout, C., Basri, G., Bouvier, J., 1987, Astrophys. J., submitted 
DIscussion

BASRI The Pis stars inhabit an unfortunate regine of rotations. They are mostly too slow for good Doppler imaging ( 10 - 25 $\mathbf{k w} / \mathrm{s}$ ) and mostly too fast for measurement of magnetic line broadening (also $10-25 \mathrm{~km} / \mathrm{s}$ ). It will therefore be difficult to pursue either of these techniques with them, though certainly worthwile if successful. BOUVIER There are a couple of stars rotating fast enough 70 $100 \mathrm{kw} / \mathrm{s}$ to use for Doppler Imaging, and they are also both fortunately bright.

MOROSSI How did you determine the effective temperatures of your $T$ Tauri stars ? What about spectral classification uncertainties ? BOUVIER Effective temperatures are deduced from the spectral type according to Cohen and Kuhi's (1979) Sp-Teff scale. Uncertainties on the spectral type are of the order of 1 or 2 subclasses. 